\title{
Aplicación de las TICs en el sector educativo: una revisión de la literatura científica de los últimos 5 años
}

\section{Application of the ICTs in the educational sector: a review of the scientific literature of the last 5 years}

\author{
Bryan Axel Gutiérrez-Palomino ${ }^{11}$, Steven Brandhon Ludeña-Gavino ${ }^{2}$, \\ Rosalynn Ornella Flores-Castañeda ${ }^{3}$, María Eudelia Acuña-Meléndez ${ }^{4}$; \\ Sandro Olaya-Cotera ${ }^{5}$, Elba María Andrade-Díaz ${ }^{6}$ \\ Universidad Privada del Norte, Lima, Perú ${ }^{123}$ \\ Universidad Nacional Federico Villarreal, Lima, Perú ${ }^{4}$ \\ Universidad San Ignacio de Loyola, Lima, Perú ${ }^{56}$ \\ (iD) ORCID ID: https://orcid.org/0000-0002-4870-4539 1 \\ (iD) ORCID ID: https://orcid.org/0000-0003-3433-8010² \\ iD ORCID ID: https://orcid.org/0000-0002-5573-359X ${ }^{3}$ \\ iD ORCID ID: https://orcid.org/0000-0002-5188-3806 \\ (iD) ORCID ID: https://orcid.org/0000-0003-4309-568X \\ (iD) ORCID ID: https://orcid.org/0000-0002-5025-213X
}

Recibido: 02 de junio de 2021

Aceptado: 21 de setiembre de 2021

\begin{abstract}
Resumen
El objetivo de la presente investigación es analizar y recopilar la información de los estudios teóricos y empíricos sobre la aplicación de las TICs en el sector educativo en los últimos 5 años. En esta revisión sistemática, se consideraron 61 artículos, de los cuales solo 30 se encuentran alineados con el objetivo planteado. Asimismo, se hace mención que existen más de 10 bases de datos con acceso a este tema; sin embargo, cierto número de ellas presentan algunas limitaciones, en cuanto a su enfoque y aplicación. No obstante, se hizo uso solamente algunas de ellas, las cuales fueron Proquest Central, Scielo, Redalyc, Dialnet y Ebsco (Fuente Académica Premier, Education Source, Complementary Index, OpenAIRE y Open Access Journals). En los resultados se estableció que la aplicación de TICs en el sector educativo es un enfoque prometedor, porque tanto los estudiantes como la plana docente que utilizan estos instrumentos tecnológicos tienen una cierta ventaja sobre aquellos que no. Se concluye que las TICs han revolucionado al ámbito de la educación de manera favorable, cambiando por completo la metodología de enseñanza tradicional; debido a esto, hoy en día existen escenarios en los cuales la plana docente y el alumnado trabajan haciendo uso de estos medios, creando así competencias tecnológicas.
\end{abstract}

${ }^{1}$ Correspondencia al autor

E-mail: bryan36ab@gmail.com 
Palabras claves: TICs, sector educativo, tecnologías de la información y la comunicación, educación, base de datos.

\begin{abstract}
The objective of this research is to analyze and compile information from theoretical and empirical studies on the application of ICTs in the education sector in the last 5 years. In this systematic review, 61 articles were considered, of which only 30 are aligned with the objective set. Likewise, it is mentioned that there are more than 10 databases, with access to this topic. However, some of them have some limitations, such as focus and application. Though only some of them were used, which were Proquest Central, Scielo, Redalyc, Dialnet and Ebsco (Premier Academic Source, Education Source, Complementary Index, OpenAIRE and Open Access Journals). The results established that the application of ICTs in the education sector is a promising approach, because both the students and the teaching staff who use these technological instruments have a certain advantage over those who do not. It is concluded that ICTs have revolutionized the field of education in a favorable way, completely changing the traditional teaching methodology, due to this, today there are scenarios in which the teaching staff and the students work using these means, thus creating technological competencies.
\end{abstract}

Keywords: ICTs, education sector, information and communication technologies, education, databases.

\title{
Introducción
}

Actualmente, se vive una época donde las tecnologías de la información y comunicación -también conocidas como TICs - avanzan de forma exponencial. Esto conlleva a que todo ámbito de nuestra vida se vea influenciada por ellas. El ejemplo más claro que se puede visualizar es en la medicina, que con el uso de software se puede detectar y prevenir enfermedades de manera temprana (Levano-Francia et al., 2019). El sector educativo no es indiferente, porque año tras año, se crean y aplican metodologías auxiliadas por la tecnología que promueven el aprendizaje del estudiante. Para Hernández, Orrego y Quiñones (2018) no es desconocido que, con la aparición del internet, se han originado múltiples cambios en la forma de cómo obtener la información; y ello ha conllevado, sin lugar a duda, a que las personas se vean en la necesidad de aprender a interactuar con las distintas herramientas tecnológicas. En este caso, Sánchez-García y Toledo-Morales (2018) confirman que las TICs forman parte de nuestra vida, de manera que es una necesidad que se implementen en las escuelas, a fin que se pueda tener una buena formación.

Por otro lado, hoy en día las TICs se han incrustado de forma progresiva en el sector educacional, de manera que la mayor parte de la enseñanza metodológica tiene sus cimientos en implementar dichas herramientas; sin embargo, existe un sector poblacional que no puede acceder a estos servicios por diferentes circunstancias, ya sea social, económico, geográfico, 
etc. Según Castro (2018), existe una gran cantidad de alumnado que es indiferente a la disponibilidad de las TICs, que pueden ser viables para su época estudiantil. Por ello, la llegada de las TICs ha abierto nuevas maneras de direccionar la educación; en consecuencia, las instituciones educativas están implementando revolucionarias metodologías al momento de impartir enseñanza, con el uso de dispositivos o herramientas como tablets, celulares, laptops, etc. Ante esto, Martínez y Duart (2016) plantean que, gracias a estos cambios, la enseñanza y el aprendizaje tradicional se han dejado de lado por la limitación geográfica.

Asimismo, Otero et al. (2019) mencionan que el uso de plataformas en línea y e-learning son utilizadas hoy en día para una mejor calidad de enseñanza. En ese sentido, Claudemir (2018) menciona que, año tras año, se implementan metodologías especiales enfocadas a todo tipo de grupo poblacional. Gracias a ello, el conocimiento que se genera por el uso de dichas herramientas será en bien de la sociedad. Según Hernández, Orrego y Quiñones (2018), el docente es quien aún en estos tiempos de revolución tecnológica conduce el proceso de enseñanza; sin embargo, no todos ellos son conscientes de que primero deben aprender a usar de una forma eficiente y eficaz los aparatos tecnológicos para integrarlos en la educación.

Para Suasnabas et al. (2017), en el proceso formativo donde se aplican las TICs se debe dar relevancia a tres aspectos cruciales para obtener los resultados esperados: el contenido que se va a compartir con los estudiantes, la infraestructura tecnológica y el conocimiento del docente. Sin lugar, a duda uno de estos aspectos involucra que el docente se actualice constantemente de tal forma que innove en la forma de brindar sus clases, en donde el estudiante se sienta atraído y motivado por aprender (Claudemir, 2018); es decir, el docente debe tener la capacidad de aprender y fortalecer sus competencias digitales para poder enseñar a los estudiantes y que ello represente una familiarización con el uso de las TICS. Sí una institución educativa opta por la implementación y uso de los dispositivos tecnológicos no implica que la calidad en el proceso de formativo esté garantizada. Antes bien, ello será el fruto de un proceso holístico (Hung, Sartori \& Marcano, 2019).

Debido a los cambios que se siguen dando en el ámbito tecnológico, los docentes y estudiantes tienen que exigirse una mayor preparación para que se adapten. Esto involucra que las instituciones educativas velen no solo por el hardware, sino también desarrollen programas que busquen eliminar las brechas digitales donde el estudiante tenga un enfoque constructivista y el rol de docente sea un facilitador (Maldonado, 2018). A esto, añade Castro (2018) que gran parte de los estudiantes recurren a la tecnología para acceder a sus redes sociales más que por temas académicos o profesionales; es decir, no tienen conocimiento de los dispositivos tecnológicos que podrían utilizar para su proceso de aprendizaje. Por ello, ante la situación 
actual, el presente artículo tiene como objetivo analizar y recopilar la información de los estudios teóricos y empíricos sobre la aplicación de las TICs en el sector educativo a partir de la revisión de diversas publicaciones de los últimos 5 años.

\section{Metodología}

De acuerdo al objetivo propuesto, se desarrolló una revisión sistemática de 61 artículos científicos. En cuanto a los criterios de elección de las bases de datos, se propusieron dos: aquellos que se encuentren en artículos científicos cuyo contenido se relacione con la aplicación de las TICS en el sector educativo en los últimos 5 años y el acceso que tenemos a bases de datos como: Proquest Central, Scielo, Redalyc, Dialnet y Ebsco (Fuente Académica Premier, Education Source, Complementary Index, OpenAIRE y Open Access Journals) sumando 30 artículos.

Los criterios de inclusión considerados fueron: que el artículo esté alineado con el objetivo de investigación planteado, que el año de publicación no supere a los 5 años y la comprobación de la validez del estudio. En referencia a los criterios de exclusión se consideró: que el año de publicación sea superior a los 5 años, si la investigación no tiene relación con el objetivo principal de la misma y si hubiera duplicidad de investigaciones.

Para el estudio se utilizaron las siguientes sentencias booleanas: Como primera instancia se indagó en Proquest Central con las sentencias, ("TICS" OR "tecnología de información y comunicación") AND (“educación” OR "sector educativo"). En Scielo (“TICS”) AND (“educación”). Asimismo, se buscó en Redalyc, utilizando (“TICS en la educación”), en Ebsco (“TICS”) AND (“educación”) y finalmente en Dialnet como (“TICS en el sector educacional”).

\section{Resultados}

La recolección de artículos se realizó a partir de múltiples bases de datos (tabla 1) haciendo un total de 61. En el transcurso, se encontraron investigaciones que se alejaban del objetivo formulado, por lo que fueron descartados; otros hablaban de TICs pero aplicados a 
sectores que no pertenecían al entorno educacional, dando un total de 30 artículos, los cuales cumplieron con los estándares de selección.

\section{Tabla 1}

Artículos encontrados e incluidos

\begin{tabular}{lcc}
\hline \multicolumn{1}{c}{ Base de datos } & $\begin{array}{c}\text { Artículos } \\
\text { encontrados }\end{array}$ & $\begin{array}{c}\text { Artículos } \\
\text { incluidos }\end{array}$ \\
\hline Dialnet & 5 & 2 \\
ProQuest Central & 18 & 9 \\
Redalyc & 15 & 6 \\
Scielo & 13 & 7 \\
Ebsco (Fuente Académica Premier, Education & 10 & 6 \\
Source, Complementary Index, OpenAIRE y & & \\
Open Access Journals) & & $\mathbf{3 0}$ \\
Total & $\mathbf{6 1}$ & \\
\hline
\end{tabular}

Fuente: Elaborado por los autores

En la tabla 2 se muestra el listado de artículos considerados en este estudio 
Tabla 2

Listado de artículos considerados en el estudio

\begin{tabular}{lll}
\hline $\mathbf{N}^{\mathbf{o}}$ & \multicolumn{1}{c}{ Autores } & \multicolumn{1}{c}{ Título } \\
\hline 1 & Acosta et. al. & $\begin{array}{l}\text { El uso del tic como recurso para el desarrollo de habilidades de } \\
\text { razonamiento lógico en estudiantes universitarios } \\
\text { Tic para la educación: sistema adaptativo basado en mecanismos de } \\
\text { aprendizaje automático para la apropiación de tecnologías en } \\
\text { estudiantes de educación media }\end{array}$ \\
2 & Otero et. al & $\begin{array}{l}\text { Impacto de las TIC en la educación: retos y perspectivas } \\
\text { El profesor y el uso de la tecnología en el proceso de enseñanza y } \\
\text { aprendizaje }\end{array}$ \\
4 & $\begin{array}{l}\text { Hernandez Ronal Mandemir Públio } \\
\text { Júnior }\end{array}$ & $\begin{array}{l}\text { La implementación de tic y mdd en la práctica docente de educación } \\
\text { primaria }\end{array}$
\end{tabular}

$6 \quad$ Morales et al.

Las tics en la educación intercultural

7 García Tormo F. Vicente

8 Hernández et al.

9 Dominguez Hilda

López, Carmona

Vazquez Héctor

10 Hung et al.

11 Plaza de la Hoz Jesús

Aplicación de tics (formularios on-line) como metodología docente activa en estudios de postgrado

Nuevas formas de aprender: la formación docente frente al uso de las tic

El uso de las tic y sus implicaciones en el rendimiento de los alumnos de bachillerato. Un primer acercamiento

Factores que inciden en el aprovechamiento de las tic de docentes colombianos/as

\section{Sánchez-García José, Toledo-Morales P. \\ 13 Suasnabas et al.}

14 Sánchez-Otero et al.

15 Maldonado María Eugenia

16 Alcalá María Jesús Balboa

17 Lamos Linares María

18 Marín et al.

19 Molina et al.

20 Reyes Chávez Rafael, Prado Rodríguez

Anna

21 Mendoza Villamar R.; Tecnologías de la información y las comunicaciones más utilizadas Quiroz Valencia Patricio
Ventajas y desventajas del uso adolescente de las tic: visión de los estudiantes

Uso de las tecnologías de la información y comunicación en la enseñanza de historia

Las tics en los procesos de enseñanza y aprendizaje en la educación universitaria

Estrategias pedagógicas en procesos de enseñanza y aprendizaje en la educación superior incluyendo tecnologías de la información y las comunicaciones

El aula, espacio propicio para el fortalecimiento de competencias ciudadanas y tecnológicas

El mundo de la tecnología especial: las tics en la educación especial

Mitos y realidades del uso de las tic en la enseñanza del inglés con fines específicos

La realidad aumentada en educación primaria desde la visión de los estudiantes

Las tic en escuelas rurales: realidades y proyección para la integración

Las tecnologías de información y comunicación como herramienta para una educación primaria inclusiva por universitarios 


\begin{tabular}{|c|c|c|}
\hline 22 & $\begin{array}{l}\text { Castro Tesén Rosa } \\
\text { Dolores }\end{array}$ & $\begin{array}{l}\text { Manejo de tecnología e información científica en la formación } \\
\text { universitaria }\end{array}$ \\
\hline 23 & Peláez et al. & $\begin{array}{l}\text { Las tics y el uso de evea en instituciones de educación básica en } \\
\text { guayaquil-ecuador }\end{array}$ \\
\hline 24 & Sandia et al. & $\begin{array}{l}\text { Apropiación de las tecnologías de información y comunicación } \\
\text { como generadoras de innovaciones educativas }\end{array}$ \\
\hline 25 & $\begin{array}{l}\text { Varela-Ordorica } \\
\text { Sandra, Valenzuela- } \\
\text { González }\end{array}$ & $\begin{array}{l}\text { Uso de las tecnologías de la información y la comunicación como } \\
\text { competencia transversal en la formación inicial de docentes }\end{array}$ \\
\hline 26 & $\begin{array}{l}\text { Zhizhko, Elena } \\
\text { Anatolievna }\end{array}$ & $\begin{array}{l}\text { Las tic y tutoría virtual en la educación de personas jóvenes y } \\
\text { adultas en México }\end{array}$ \\
\hline 27 & Sánchez et al. & $\begin{array}{l}\text { La formación de docentes normalistas: de la tradición pedagógica a } \\
\text { los entornos virtuales de aprendizaje }\end{array}$ \\
\hline 28 & Cariaga, Romina & Experiencias en el uso de las tic. Análisis de relatos de docentes \\
\hline 29 & Aguiar et al. & $\begin{array}{l}\text { Cambios y permanencias en las prácticas de enseñanza con tic, } \\
\text { neuquén, argentina }\end{array}$ \\
\hline 30 & $\begin{array}{l}\text { Fernandez Zalazar } \\
\text { Diana, Soto Romina } \\
\text { Jodre, }\end{array}$ & Prácticas docentes y tic en el nivel superior \\
\hline
\end{tabular}

Fuente: Elaborado por los autores

En la tabla 3 se muestra las investigaciones realizadas por países.

Tabla 3

Investigaciones por países

\begin{tabular}{lcc}
\hline \multicolumn{1}{c}{ País } & $\mathbf{N}^{\mathbf{0}}$ de artículos & Porcentaje \\
\hline Colombia & 8 & $26.67 \%$ \\
Ecuador & 5 & $16.67 \%$ \\
España & 4 & $13.33 \%$ \\
México & 4 & $13.33 \%$ \\
Argentina & 3 & $10.00 \%$ \\
Costa Rica & 2 & $6.67 \%$ \\
Chile & 1 & $3.33 \%$ \\
Brasil & 1 & $3.33 \%$ \\
Perú & 1 & $3.33 \%$ \\
Venezuela & 1 & $3.33 \%$ \\
\hline Total & $\mathbf{3 0}$ & $\mathbf{1 0 0 . 0 \%}$ \\
\hline
\end{tabular}

Fuente: Elaborado por los autores

Se puede apreciar a los 30 artículos de investigación, donde Colombia cuenta con 8 (26.67\%), seguido de Ecuador con 5 (16.67\%), y estos son los países con más estudios relacionados con el objetivo de esta presente investigación. Por su parte, España y México cuentan con 4 investigaciones (13.33\%), seguido de Argentina con 3 investigaciones (10.00\%), 
Costa Rica con 2 investigaciones (6.67\%), y Chile, Brasil, Perú y Venezuela con una investigación (3.33\%). En la tabla 4 se muestra los tipos de investigaciones realizadas.

\section{Tabla 4}

Tipo de investigación

\begin{tabular}{lcc}
\multicolumn{1}{c}{ Tipo } & $\mathbf{N}^{\mathbf{0}}$ de artículos & Porcentaje \\
\hline Cualitativa & 17 & $56.67 \%$ \\
Mixta & 9 & $30.00 \%$ \\
Cuantitativa & 4 & $13.33 \%$ \\
\hline Total & 30 & $100.0 \%$
\end{tabular}

Fuente: Elaborado por los autores

Se evidencia que en las investigaciones analizadas predomina tipo de investigación cualitativa con 17 (56.67\%) artículos. Esto demuestra que, al momento de llevar a cabo un trabajo se espera tener un resultado a partir de respuestas libres para su posterior interpretación de significados. La cuantitativa con $4(13.33 \%)$ artículos, son investigaciones que buscan reflejar la información recolectada en datos o tablas.

\section{Discusión}

Para Hernandez (2017) el uso de las TIC ha logrado un impacto en la sociedad, propiciando diversos cambios que han beneficiado a muchos sectores, siendo el educativo el que mayor influencia ha tenido. En ese ámbito se han venido implementando nuevos métodos de enseñanza que van de la mano con herramientas como las plataformas virtuales. En estas los docentes cumplen un rol importante, pues deben tener la capacidad de comprenderlas y usarlas de una manera sencilla; del mismo modo, se encuentran las bases de datos, que facilitan a los estudiantes y docentes la búsqueda de información de una manera más rápida y sencilla, sin la necesidad de asistir a bibliotecas.

Para Cariaga (2018), aquellos profesores que hacen uso de la tecnología presentan cambios favorables con respecto a la impartición de clases. También existen dificultades al momento de implementarlas, comenzando por la realidad que algunos docentes no se encuentran capacitados para poder usar algunas herramientas; esto se debe a que gran parte de ellos no reciben una capacitación adecuada ante las TICs o son reacios a usarlas, optando por continuar en su metodología tradicional. 
En el caso de Varela-Ordorica y Valenzuela-González (2020), observaron que los docentes aceptaron sus limitaciones en el uso de las TICs; sin embargo, estos son conscientes sobre la importancia de su uso a fin de poder innovar en el proceso de enseñanza. Hoy en día, se puede recurrir a diversas formas para adquirir conocimientos informáticos; a fin que los estudiantes logren un aprendizaje significativo y que los docentes fortalezcan su competencia en el uso de tecnología en su labor educativa (Maldonado, 2018).

Por su parte, Molina y Mesa (2018) mencionan en su investigación realizada en Colombia, la carencia de capacitación de calidad por parte del Estado hacia los docentes en referencia al uso de las TICs. En ese sentido, el fortalecimiento de competencias digitales del docente en el uso de herramientas digitales debe ser una prioridad y, por lo tanto, debe ser incluida dentro de los programas que plantea el Estado.

Según la investigación de Suasnabas et al. (2017) en la ciudad de Ecuador, en diversas escuelas el uso de una computadora solo se limita a los directores y profesores. Ello es un problema muy recurrente en Latinoamérica, donde en muchas zonas no se tiene acceso a la tecnología. Según Otero et al. (2019), en la actualidad es una exigencia que las instituciones educativas implementen una infraestructura tecnológica, donde el docente aplique sus conocimientos para impartir sus clases a fin que el estudiante desarrolle su capacidad de concentración y aprendizaje. Esto conlleva a pensar en la situación del sector público, de aquellas escuelas en las zonas rurales en donde apenas existe un espacio físico para el dictado de clases; y es el Estado quien tiene la gran responsabilidad de lograr que la revolución tecnológica también llegue a esos lugares.

Para Castro (2018), los estudiantes que formaron parte de su investigación demostraron, al inicio, que no conocían herramientas tecnológicas tales como Prezzi y Cloud, lo cual evidencia que - aún en estos tiempos-no se conoce y/o aprovecha las TICs en el entorno educativo. Este problema es evidente no solo en estudiantes, pues el docente es uno de los principales actores en el proceso de enseñanza-aprendizaje, por lo cual es un imperativo su capacitación en el uso de las TICs y lograr desarrollar la competencia digital en los estudiantes.

En Ecuador, Mendoza y Quiroz (2019) obtuvieron como resultado de su estudio que el $64 \%$ de los estudiantes universitarios utilizan su equipo móvil para desarrollar diversas actividades, el $24 \%$ hace uso de la laptop y el $12 \%$ el ordenador personal. Esto muestra que el celular es el dispositivo portátil usado con mayor frecuencia, pues permite a su usuario tener acceso a la red en todo momento lo cual es una gran ventaja (Martínez \& Duart, 2016). 
Por todo ello, es evidente que la aplicación de TICs en el sector educativo es un enfoque prometedor, porque el alumnado y la plana docente-que están inmersos en el uso de estas herramientas tecnológicas - tienen una cierta ventaja sobre aquellos que no, lo que les permite poseer una formación sobre las clases de manera llamativa. Asimismo, los profesores adquieren nuevas habilidades para compartir el conocimiento hacia sus alumnos; es decir, el docente y el estudiante que hacen uso de las TIC las han convertido en un recurso muy importante en el proceso de enseñanza-aprendizaje (Hernandez, 2017). Por tal motivo, para Sapién et al. (2020), los estudiantes valoran mucho el acceso a la información en el mundo en su proceso de aprendizaje. Asimismo, al usar las TICs logran interactuar con otras personas y ello enriquece el proceso formativo.

Dentro de las limitaciones se encontró que no todos los docentes y estudiantes tienen la posibilidad de tener acceso a las TICs, lo cual impide que obtengan ventajas y beneficios en el proceso de enseñanza-aprendizaje. Esto lo mencionan Molina y Mesa (2018), quienes revelan que se puede utilizar medios tecnológicos para fortalecer la educación, pero que ello tiene un límite, ya que hay zonas que no cuentan con los mismos recursos que otras. Desde el punto de vista de Caro y Flores (2018), en la actualidad aún existen diversas insuficiencias en el desarrollo de programas educativos relacionados con el uso de las TICs, a pesar de que ello signifique una gran oportunidad para lograr obtener información relevante en el momento de tomar decisiones, buscando siempre alcanzar la calidad educativa. Finalmente, para Sapién et al. (2020) uno de los grandes inconvenientes que existe es la velocidad de conexión del internet, la cual impide que el estudiante no pueda acceder a sus clases o a buscar información sin interrupciones.

\section{Conclusiones}

La presente revisión sistemática contiene un total de 30 artículos que guardan relación con la aplicación de las TICs en el sector educativo. En los últimos 5 años, las TICs han revolucionado al ámbito de la educación de manera favorable, cambiando por completo la metodología de enseñanza tradicional, y debido a esto, existen escenarios en los cuales la plana docente y el alumnado trabajan haciendo uso de estos medios. La resultante de los profesores luego de aplicar las TICS, han dado cambios favorables al momento de implementarlas, otra realidad es que algunos presentaron dificultades para poder entender el funcionamiento.

Hoy más que nunca es prioritario el uso de la tecnología para innovar el proceso formativo con el objetivo de modernizarlo, y ello sin lugar a duda tiene como participantes a docentes y estudiantes. Se afirma, entonces, que en el pasado debe quedar lo tradicional y 
empezar a usar las TICs, las cuales brindan herramientas de apoyo tecnológicas para lograr desarrollar las competencias educativas.

\section{Referencias}

Acosta, Z., Jimenez, O., Toscano, J., Luzuriaga, E. \& Urrea, H. (2019). El uso de las tic como recurso para el desarrollo de habilidades de razonamiento lógico en estudiantes universitarios. Revista Ibérica de Sistemas e Tecnologías de Informação, 200-209. https://search.proquest.com/docview/2258687293?accountid=36937

Aguiar, D., Capuano, A., Diez, M., Fourés, C. \& Silin, I. (2016). Cambios y permanencias en las prácticas de enseñanza con tic, Neuquén, Argentina. Ciencia, docencia y tecnología,

http://www.scielo.org.ar/scielo.php?script=sci_arttext\&pid=S18511716201600020001 $3 \& \operatorname{lng}=\mathrm{es} \& \operatorname{tlng}=\mathrm{es}$

Alcalá, M. (2016). El mundo de la tecnología especial: las tics en la educación especial. Revista Internacional de apoyo a la inclusión, logopedia, sociedad y multiculturalidad, 2 (2),97-105. https://www.redalyc.org/articulo.oa?id=5746/574660898008

Cariaga, R. (2018). Experiencias en el uso de las tic: análisis de relatos de docentes. Ciencia, docencia $y$

tecnología,

(56),

131-155.

http://www.scielo.org.ar/scielo.php?script=sci_arttext\&pid=S1851-

$17162018000100006 \& \operatorname{lng}=$ es\&tlng=es.

Caro, L. \& Flores, N. (2018). Programas educativos con uso de tic en la región Bogotá Cundinamarca-Colombia: un modelo de evaluación. Edmetic, 7 (1), 297-320. http://dx.doi.org/10.21071/edmetic.v7i1.6746

Castro, R. (2018). Manejo de tecnología e información científica en la formación universitaria. $\begin{array}{lllll}\text { Inclusión } \quad \& \quad \text { Desarrollo, } & 5 & \text { (2), }\end{array}$ http://dx.doi.org/10.26620/uniminuto.inclusion.5.2.2018.63-82

Claudemir, J. (2018). El profesor y el uso de la tecnología en el proceso de enseñanza y aprendizaje. Revista Ibero-Americana de Estudos em Educação, 13 (3), 1092-1105. http://dx.doi.org/10.21723/riaee.v13.n3.2018.11190

Fernández, D., Jofre, C. \& Soto, R. (2016). Prácticas docentes y tic en el nivel superior. Anuario $\begin{array}{llll}\text { de } & \text { investigaciones, } & 23 & (1),\end{array}$ http://www.scielo.org.ar/scielo.php?script=sci_arttext\&pid=S1851$16862016000100010 \& \operatorname{lng}=$ es\&tlng=es. 
García, F. (2018). Aplicación de tics (formularios on-line) como metodología docente activa en estudios de postgrado. International Journal of Developmental and Educational Psychology, 3 (1),199-207. https://www.redalyc.org/articulo.oa?id=3498/349856428020

Hernandez, R. (2017). Impacto de las tic en la educación: retos y perspectivas/ impact of ICT on education: challenges and perspectives. Propositos y Representaciones, 5 (1), 325347. http://dx.doi.org/10.20511/pyr2017.v5n1.149

Hernández, R., Orrego, R. \& Quiñones, S. (2018). Nuevas formas de aprender: la formación docente en el uso de las tic. Propósitos y Representaciones 6 (2), 671-701. http://dx.doi.org/10.20511/pyr2018.v6n2.248

Hung, E., Sartori, A. \& Marcano, B. (2019). Factores que inciden en el aprovechamiento de las tic de docentes colombianos/as. Prisma Social, (25), 464-487. https://search.proquest.com/docview/2248268645 accountid=36937

Lamos, M. (2019). Mitos y realidades del uso de las tic en la enseñanza del inglés con fines específicos. Revista de Lenguas Modernas, 30, 2019. http://search.ebscohost.com/login.aspx?direct=true\&db=edsdoj\&AN=edsdoj.7898561 fe6c64869bf2375bd727d2177\&lang=es\&site=eds-live

Levano-Francia, L. et al. (2019). Competencias digitales y educación. Propósitos y Representaciones, https://search.proquest.com/docview/2253100294/fulltextPDF/DBF31963C4834D7D $\mathrm{PQ} / 1$ ?accountid=36937

Maldonado, M. (2018). El aula, espacio propicio para el fortalecimiento de competencias $\begin{array}{llllll}\text { ciudadanas } & \text { y } & \text { tecnológicas. } & \text { Sophia, } & 14 & \text { (1), }\end{array}$ https://www.redalyc.org/articulo.oa?id=4137/413755833004

Marín, V. \& Sampedro-Requena, B. (2020). La realidad aumentada en educación primaria desde la visión de los estudiantes. Alteridad, 15 (1), 61-73. http://dx.doi.org/10.17163/alt.v15n1.2020.05

Martínez, R. \& Duart, J. (2016). Nuevas tendencias de aprendizaje colaborativo en e-learning. Claves para implementación efectiva. https://www.researchgate.net/publication/307601105_Nuevas_tendencias_de_aprendi zaje_colaborativo_en_e-learning_Claves_para_su_implementacion_efectiva

Mato, D. \& Álvarez, D. (2019). La implementación de tic y mdd en la práctica docente de $\begin{array}{lllll}\text { educación } & \text { primaria. } & \text { Campus } & \text { Virtuales, } 8 & \text { (2), }\end{array}$ 
http://search.ebscohost.com/login.aspx ?direct=true\&db=eue\&AN=139557141\&lang= es\&site=eds-live

Mendoza, R. \& Quiroz, P. (2019). Tecnologías de la información y las comunicaciones más $\begin{array}{lllllll}\text { utilizadas por } & \text { universitarios. } & 3 c & \text { Tic, } & 8 & \text { (4), } & \text { 27-43. }\end{array}$ http://dx.doi.org/10.17993/3ctic.2019.84.27-43

Molina, L. \& Mesa, F. (2018). Las tic en escuelas rurales: realidades y proyección para la $\begin{array}{llllll}\text { integración. } & \text { Praxis } & \& & \text { Saber, } & 9 & \text { (21), }\end{array}$ http://dx.doi.org/10.19053/22160159.v9.n21.2018.8924

Morales, E., Morales, E. \& Chiluisa, J. (2017). Las tics en la educación intercultural. Revista Publicando. https://dialnet.unirioja.es/servlet/articulo?codigo=6115191

Otero, A., Rivera, W., Pedraza, C. \& Canay, J. (2019). Tic para la educación: sistema adaptativo basado en mecanismos de aprendizaje automático para la apropiación de tecnologías en estudiantes de educación media. Revista Telos, 21 (3), 526-543. https://doi.org/10.36390/telos213.03

Peláez, R., Morales, J., Lara, C. \& Tumbaco, M. (2018). Las TICs y el uso de evea en instituciones de educación básica en Guayaquil-Ecuador. Revista Lasallista de Investigación, 15

(2),131-140. https://www.redalyc.org/articulo.oa?id=695/69559233011

Plaza de la Hoz, J. (2017). Benefits and drawbacks of adolescent ICT use: the students' point of view; ventajas y desventajas del uso adolescente de las tic: visión de los estudiantes. Revista Complutense de Educación. https://doi.org/10.5209/RCED.53428

Reyes, R. \& Prado, A. (2020). Las tecnologías de información y comunicación como herramienta para una educación primaria inclusiva. Revista Educación, 44 (2). https://www.redalyc.org/articulo.oa?id=440/44062184011

Sánchez, J., González, A. \& Monroy, A. (2019). La formación de docentes normalistas: de la tradición pedagógica a los entornos virtuales de aprendizaje. RIDE Revista Iberoamericana para la Investigación y el Desarrollo Educativo, 10 (19). https://doi.org/10.23913/ride.v10i19.539

Sánchez-García, J. y Toledo-Morales, P. (2018). Uso de las tecnologías de la información y comunicación en la enseñanza de historia. Aposta Revista de Ciencias Sociales, 78, 8-32. http://apostadigital.com/revistav3/hemeroteca/ptoledo.pdf

Sánchez-Otero, M., García-Guiliany, J., Steffens-Sanabria, E. \& Hernández, H. (2019). Estrategias pedagógicas en procesos de enseñanza y aprendizaje en la educación 
superior incluyendo tecnologías de la información y las comunicaciones. Información tecnológica, 30 (3), 277-286. https://dx.doi.org/10.4067/S0718-07642019000300277

Sandia, B., Luzardo, M. \& Aguilar-Jiménez, A. (2019). Apropiación de las tecnologías de información y comunicación como generadoras de innovaciones educativas. Ciencia, $\begin{array}{llll}\text { docencia } & y & \text { tecnología, } & \text { 267-289. }\end{array}$ http://www.scielo.org.ar/scielo.php?script=sci_arttext\&pid=S1851$17162019000100010 \& \operatorname{lng}=\mathrm{es} \&$ tlng=es.

Sapién, A., Piñón, L., Gutiérrez, M. \& Bordas, J. (2020). La Educación superior durante la contingencia sanitaria covid-19: Uso de las tic como herramientas de aprendizaje. Caso de estudio: alumnos de la Facultad de Contaduría y Administración. Revista Latina de Comunicación Social, 78, 309-328. https://doi.org/10.4185/RLCS-2020-1479

Suasnabas, L., Avila, W., Chong, E. \& Rodriguez, V. (2017). Las tics en los procesos de enseñanza y aprendizaje en la educación universitaria. Revista Científica Dominio de las ciencias. https://dialnet.unirioja.es/servlet/articulo?codigo $=6326781$

Varela-Ordorica, S. \& Valenzuela-González, J. (2020). Uso de las tecnologías de la información y la comunicación como competencia transversal en la formación inicial de docentes. Revista Electrónica Educare, 24 (1), 172-191. https://dx.doi.org/10.15359/ree.24-1.10

Zhizhko, E. (2018). Las tic y tutoría virtual en la educación de personas jóvenes y adultas en méxico. Voces De La Educación, $3 \quad$ (6), 204-217. https://search.proquest.com/docview/2189573264?accountid=36937 